\section{Prescribing patterns and perceptions of health care professionals about rational drug use in a specialist hospital clinic}

\author{
Moses A. Ojo, ${ }^{1}$ Cecilia I. Igwilo, \\ Thomas Emedoh ${ }^{1}$ \\ ${ }^{1}$ Federal Neuro-Psychiatric Hospital Yaba, \\ Lagos; ${ }^{2}$ University of Lagos, Lagos, \\ Nigeria
}

\begin{abstract}
Irrational drug use is associated with adverse consequences including drug resistance and avoidable adverse drug reactions. Studies of rational drug use in psychiatric facilities are scanty. This study evaluated prescription practices and perception of health care professionals regarding causes of irrational drug use. A retrospective study conducted at the outpatient clinic of Federal Neuropsychiatric Hospital, Yaba, Lagos. Data on drug use indicators were analyzed. A cross-sectional assessment of perception of prescribers and dispensers regarding rational drug use was conducted. A total of 600 prescriptions were analyzed. Mean number of drugs per encounter was 3.5 and percentage generic prescribed was $58.5 \%$. Poly-pharmacy $(\mathrm{P}=0.024$, 95\% $\mathrm{CI}=1.082-1.315)$ and non-generic prescribing $(\mathrm{P}=0.032,95 \% \mathrm{CI}=1.495-1.821)$ were significantly associated with young prescribers. Factors associated with irrational drug use include demand from patients, patients' beliefs about injection drugs and influence of pharmaceutical sale representatives. Certain aspect of prescribers indicators are still poor in the hospital studied. Health care professionals identified possible associated factors for irrational drug use. Concerted efforts are required to ensure rational drug use especially in psychiatric facilities in Nigeria.
\end{abstract}

\section{Introduction}

Rational drug use refers to patients receiving medications appropriate to their clinical needs, in doses that meet their individual requirements, for an adequate period of time and at low cost to them and their community. 1,2 Access to quality, affordable, safe and effective drugs is an important component of an efficient health care system. ${ }^{3}$ Although about twothirds of the world population has regular access to medications, more than half of medical prescriptions are inappropriate. ${ }^{4}$ In addition, among those who receive appropriate prescriptions, more than half fail to take the drugs correctly.

Data on irrational drug use has shown an increasing trend worldwide leading to adverse health consequences. Factors associated with irrational drug use include poor knowledge of medical staff, short years of service, traditional beliefs about illness, short consultation time, lack of interaction between dispenser and patients and poor implementation of the National Drug Policy guidelines. ${ }^{3,4,6,7}$ Studies in psychiatric settings have identified associated factors such as influence of institutional culture on prescribing patterns, low staff-topatient ratios, poor level of non-medical treatments, and poor diagnosis. ${ }^{8,9}$

Adverse effects of irrational drug use are enormous. These include wastage of resources, transmission of infections through inappropriate use of injections, increase in resistance to anti-malarial and anti-microbial drugs and increase in drug-related morbidity and mortality. ${ }^{4,5,7}$ Although rational drug use is an essential part of National Drug Policy, reports from WHO have suggested that efforts to promote rational drug use are poor or nonexistent in many developing countries. Local researches on this topic are also few and farbetween. Thus, it has become imperative to examine drug use practices of hospitals in Nigeria regarding compliance with the principles of rational drug use.

The aim of this study is to evaluate drug use practices and application of rational use principles among prescribers and dispensers in the hospital. It also aimed to assess the perception of prescribers and dispensers regarding factors associated with rational use of drugs.

\section{Materials and Methods}

The study was carried out at the out-patient clinic of the Federal Neuro-Psychiatric Hospital, Yaba, Lagos. The study designs were in two parts including a retrospective assessment of medical prescriptions in the last 12 months and a cross-sectional study of the perception of health care professionals regarding rational drug use.

\section{Ethical considerations}

The ethical review committee of the hospital approved the study. Written informed consent was obtained from respondents after the purpose of the study was explained to them.

\section{Sample size}

The WHO recommends at least 600 prescrip-
Correspondence: Moses A. 0jo, Federal NeuroPsychiatric Hospital Yaba, Lagos PMB 2008, Yaba Lagos, Nigeria.

Tel.: 08033652478.

E-mail: ojoabayomimoses@yahoo.co.uk

Acknowledgments: hospital management, Federal Neuro-psychiatric Hospital Yaba, Lagos, Nigeria.

Key words: drug use indicators, perceptions, rational use, poly-pharmacy.

Contributions: MA0, idea conception, drafting of proposal, designing, collection, analysis, and interpretation of data, drafting and revising the manuscript and final review and approval of version to be sent for publication; CII, idea conception, study design, interpretation of manuscript, revising manuscript and final approval of version to be published; TE, idea conception, study design, collection and collation of data and data analysis, revising of manuscript and review and approval of the final version for publication.

Conflict of interests: the authors declare no potential conflict of interests.

Received for publication: 11 July 2012

Revision received: 17 February 2013.

Accepted for publication: 4 February 2014.

This work is licensed under a Creative Commons Attribution NonCommercial 3.0 License (CC BYNC 3.0).

(C) Copyright M.A. Ojo et al., 2014

Licensee PAGEPress, Italy

Journal of Public Health in Africa 2014; 5:242

doi:10.4081/jphia.2014.242

tions for study on drug use indicators. ${ }^{10}$ Hence, 600 prescriptions were randomly selected. The second part of the study included 50 prescribers and 46 dispensers.

\section{Instruments}

\section{WHO prescribing indicators form}

This instrument was designed to measure specific aspects of the behaviour of healthcare providers. The techniques for using the form have been well tested, and can be implemented in a standard way by individuals without special training.

\section{A self administered questionnaire}

This was used to assess the perception of prescribers and dispensers regarding rational use of drugs. It included questions on sociodemographic parameters, year of qualification, years of clinical experience, and perceived reasons for specific irrational use practices among others. 


\section{Study procedure}

A retrospective assessment of prescriptions issued in the last 12 months was carried out using systematic random sampling technique. A sample frame consisting of a list of all the encounters in the period under study was prepared. A total of 48,235 encounters were listed. The sampling interval was obtained by dividing 48,235 by 600 giving approximately 80 . Thus, after selecting the first encounter using a random sampling technique, subsequent encounters were selected at intervals of 80 till the sample size of 600 was attained.

A retrospective chart was constructed to assess socio-demographic and drug use indicators (DUIs) in the selected prescriptions.

A self-administered questionnaire was also administered to examine the attitude of health care professionals regarding rational drug use. This questionnaire contained follow-up questions from performance on the prescribing indicators.

\section{Data analysis}

Data was collated and analyzed using the Statistical Package for Social Sciences (SPSS 18). Descriptive statistics such as proportions and percentages were calculated. Test of association was carried out using Chi square with level of significant set at $\mathrm{P}<0.05$. Drug use indicators were calculated using the WHO recommended formulae.

\section{Results}

Table 1 shows DUIs in the outpatient clinic of the hospital. A total number of 2108 drugs were prescribed in 600 encounters with a mean number of drugs per encounter of 3.51 $(\mathrm{SD}=1.33)$. About one-fifth (19\%) of the prescriptions contained four or more drugs suggesting a relatively low level of poly-pharmacy. Percentage of generic drugs prescribed was $58.5 \%$ with $41.3 \%$ of prescriptions having 2 or more non-generic drugs. The percentage of prescriptions with one or more injections was $38.7 \%$. A high proportion of drugs prescribed (96.3\%) were from the essential drug list (EDL). However, there were low percentages of prescriptions with antibiotics (0.33\%) and analgesics (2\%).

Table 2 illustrates the relationship between drug use indicators (e.g. poly-pharmacy, nongeneric prescription, etc.) and professional status of prescribers. A significantly higher proportion of prescribers who prescribed four or more drugs per encounter were junior doctors with fewer years of clinical experience (59.7\%) compared to senior doctors and consultants (40.3\%) ( $\mathrm{P}=0.024,95 \% \quad \mathrm{CI}=1.082$ 1.315). Similarly, a significantly higher propor- tion of prescribers who prescribed drugs in non-generic names were junior doctors (61.3\%) compared with senior doctors (38.7\%) $(\mathrm{P}=0.032,95 \% \mathrm{CI}=1.405-1.821)$.

Assessment of the perception of doctors and pharmacists regarding factors that may influence rational drug was carried out in the second part of the study. Out of a total of 107 doctors and pharmacists in the employment of the hospital at the time of the study, 96 comprising of 50 doctors and 46 pharmacists returned the questionnaire giving a response rate of $89.7 \%$. The age of respondents ranged from 21 to 43 years with a mean of $33.1(\mathrm{SD}=6.07)$. Among the respondents, doctors were significantly older with a mean age of 36 years $(\mathrm{SD}=3.77)$ compared with pharmacists with a mean age of 29.43 years $(\mathrm{SD}=6.49) \quad(\mathrm{P}=0.0001,95 \%$ $\mathrm{CI}=4.47-8.67)$. Gender distribution showed that a higher proportion of doctors were males (66.7\%). Conversely, majority of pharmacists were females (85.7\%). The differences in gender distribution was statistically significant $(\mathrm{P}=0.0001,95 \% \mathrm{CI}=4.271-33.714)$. The mean number of years post qualification was significantly higher among doctors (6.78; $\mathrm{SD}=2.116)$ compared to pharmacists (3.71; SD=4.89) $(\mathrm{P}=0.0001,95 \% \mathrm{CI}=1.593-4.534)$. Similarly, doctors had a significantly higher number years of clinical experience with a mean of
$6.78(\mathrm{SD}=2.116)$ compared with pharmacists (2.48; $\mathrm{SD}=3.03) \quad(\mathrm{P}=0.0001,95 \% \quad \mathrm{CI}=3.256$ 5.347).

Compared to $100 \%$ among doctors, only $28.6 \%$ of pharmacists agreed that they have been having regular contact with patients during the outpatient clinic consultation. The observed difference between the two groups was clinically significant $(\mathrm{P}=0.0001,95 \%$ $\mathrm{CI}=2.17-5.646)$. Although all the respondents were aware about the concept of rational drug use, only a small proportion (Doctors, 11.1\% and Pharmacists 23.8\%) were actively receiving update information on the topic. However, this difference was not statistically significant $(\mathrm{P}=0.132)$. All prescribers (medical doctors) believed they needed more information on the concept of rational drug use compared to pharmacists (85.7\%) $(\mathrm{P}=0.004,95 \% \quad \mathrm{CI}=1.031$ 1.32).

All the respondents (100\%) believed that the average number of drug prescribed per encounter in the outpatient clinic of the hospital is high. A higher proportion (97.9\%) also believed that the use of generic name in prescriptions is low in the outpatient clinic. Similarly, all respondents admitted that there is a high percentage of injection drug use (100\%), low percentage of prescribed drug from EDL (100\%) and that dispensers (phar-

Table 1. Drug use indicators at the outpatient clinic of the Hospital.

\begin{tabular}{lc} 
Drug use indicators & Values \\
Total number of encounters & 600 \\
Total number of drugs prescribed in all encounters & 2108 \\
\hline Total number of generic drugs prescribed & 1234 \\
Total number of prescription with at least one injection drug & 232 \\
\hline Total number of drugs prescribed from essential drug list & 2032 \\
Total number of prescriptions with analgesics & 12 \\
\hline Total number of prescriptions with antibiotics & 2 \\
Average number of drugs per encounter & 3.513 \\
\hline Percentage of drugs prescribed in generic name & $58.5 \%$ \\
Percentage of encounters with injections prescribed & $38.7 \%$ \\
\hline Percentage of drugs prescribed from essential drug list & $95.8 \%$ \\
Percentage of with analgesics prescribed & $2.0 \%$ \\
\hline Percentage of encounters with antibiotics prescribed & $0.33 \%$ \\
\hline
\end{tabular}

Table 2. Relationship between drug use indicators and professional status of prescribers.

\begin{tabular}{lcccc} 
& $\begin{array}{c}\text { Status of prescribers } \\
\text { Junior }\end{array}$ & Senior & P-value & $95 \%$ CI \\
Drugg use indicators & & & \multirow{2}{*}{0.024} & $1.082-1.315$ \\
$\begin{array}{l}\text { Number of drugs per encounter } \\
\quad 1-3\end{array}$ & $200(66.2)$ & $102(33.8)$ & & \\
$\quad$ 4 and above & $178(59.7)$ & $120(40.3)$ & & \\
$\begin{array}{l}\text { Number of non-generic drugs } \\
\quad \text { Less than 2 } \\
\quad 2 \text { and above }\end{array}$ & $226(64.2)$ & $126(35.8)$ & 0.032 & $1.405-1.821$ \\
\hline
\end{tabular}


macists) were not spending enough time with their patients (100\%).

Table 3 illustrates the perception of prescribers and dispensers regarding reasons for the observed drug use indicators. Majority of these professionals (93.8\%) agreed that polypharmacy may be due to the influence of patients on prescribers. This belief was held by a higher proportion of dispensers (95.6\%) compared to prescribers (92\%). Among dispensers, $95.7 \%$ agreed prescribers were profiting from sales of prescribed drugs, $52 \%$ believed diagnoses were not usually accurate and more than a third (39.1\%) believed prescribers lack required training.

Most of the respondents agreed that low percentage of prescription in generic name can be attributed to influence of pharmaceutical representatives (97.9\%) and lack of training of prescribers (60.4\%). Compared to prescribers, a higher proportion of dispensers (95.7\%) agreed that prescribers were not well trained while almost a third (30.4\%) agreed that they lack required knowledge of generic name.

Majority of respondents agreed that high rate of injection drugs prescription is attributable to high rate of indications for injections (54.2\%), beliefs of patients which encourages injection use (87.5\%) and attitude of prescribers (79.2\%). Overall, majority of respondents agreed that reasons for lack of regular and adequate contact with patients during dispensing were high number of patients at the out-patient clinic (100\%), high volume of patients at the pharmacy on clinic days (97.9\%) and poorly designed structure of the pharmacy unit (60.4\%).

\section{Discussion}

Findings from this study show some deficiencies in prescribing indicators at the outpatient clinic of the hospital. Although the average number of drugs per encounter appears normal, a significant proportion of prescribers, especially the young and inexperienced were still prescribing more than four drugs per encounter. Data analysis showed that prescribers recommended 4 or more drugs in half of the encounters with patients. The average of 3.5 drugs per encounter reported in this study was similar to the reports of studies in other developing countries. While a study in Central African Republic also reported an average of 3.5 drugs per encounter, ${ }^{11}$ studies in India and Ghana have reported 3.2 and 3.6 respectively. ${ }^{12,13}$ However, an earlier study in Brazil reported $8.6,{ }^{14}$ while a more recent study in Nepal reported 2.9 drugs per encounter. ${ }^{3}$

Poly-pharmacy has been described as the use of large number of medications or the use of potentially inappropriate medications which can increase the risk of adverse drug reactions. ${ }^{15}$ A more concise definition refers to poly-pharmacy as the practice of prescribing four or more medications to the same person. ${ }^{16}$ It is an essential drug use indicator and a measure of rational drug use. Poly-pharmacy has been identified as a major factor in increased occurrence of adverse drug reactions, severe drug-drug interaction, nonadherence to prescription and prolonged hospitalization. ${ }^{17}$ Polypharmacy has been associated with treatment of chronic medical conditions such as cardiovascular disease, diabetes and psychiatric disorders. Such conditions require multiple drugs use for prolonged period of time. Other risk factors associated with poly-pharmacy include old age, low level of awareness about medications among patients, multiple coexisting medical conditions, recent hospitalizations, and multiplicity of health care providers. ${ }^{18,19}$ Results from the present study also suggest that physicians with fewer years of clinical experience are more likely to prescribe higher number of medications per encounter.

Analysis of the selected prescriptions indicated that more than half of the drugs (58\%) were prescribed in generic name. This finding is similar to reports from the study conducted in Ghana which reported a rate of $61.1 \%{ }^{13}$ The reason for the moderately high level of generic prescription can be attributed to the extensive use of conventional antipsychotics especially the injections. Over one-third of encounters had at least one injection drug in the prescrip-

Table 3. Perception of prescribers and dispensers about drug use indicators.

\begin{tabular}{|c|c|c|c|}
\hline Drug use indicators & Prescribers N (\%) 50 & Dispensers N (\%) 46 & Total N (\%) 96 \\
\hline \multicolumn{4}{|l|}{ High number of drugs per encounter } \\
\hline Patients sometimes influence prescription & $46(92)$ & $44(95.6)$ & $90(93.8)$ \\
\hline Prescribers are profiting from sales of drugs & $2(4)$ & $44(95.7)$ & $46(47.9)$ \\
\hline Diagnoses of prescribers not usually accurate & $2(4)$ & $24(52.2)$ & $26(27.1)$ \\
\hline Prescribers lack training to prescribe accurately & $0(0)$ & $18(39.1)$ & $18(18.8)$ \\
\hline Poly-pharmacy is due to lack of drugs & $1(2)$ & $15(32.6)$ & $16(16.7)$ \\
\hline Drug Revolving Fund influence prescribers & $8(16)$ & $2(4.3)$ & $10(10.4)$ \\
\hline \multicolumn{4}{|l|}{ Low percentage of prescription in generic } \\
\hline Most drugs in the hospital are branded & $15(30)$ & $13(28.3)$ & $28(29.2)$ \\
\hline Many prescribers not well trained in using generic name & $14(28)$ & $44(95.7)$ & $58(60.4)$ \\
\hline Pharmaceutical reps influence prescription of branded drugs & $49(98)$ & $45(97.8)$ & $94(97.9)$ \\
\hline Most prescribed generic drugs are not available & $12(24)$ & $2(4.3)$ & $14(14.6$ \\
\hline Few indications exist for available generic drugs & $5(10)$ & $1(2.2)$ & $6(6.2)$ \\
\hline Most prescribers lack knowledge of generic name of drugs & $6(12)$ & $14(30.4)$ & $20(20.8)$ \\
\hline \multicolumn{4}{|l|}{ High rate of injection drugs prescription } \\
\hline Indications for injection drugs use is high & $45(90)$ & $7(15.2)$ & $52(54.2)$ \\
\hline Beliefs of patients increase prescription of injections & $42(84)$ & $42(91.3)$ & $84(87.5)$ \\
\hline Use of injections is most cost effective for patients & $6(12)$ & $4(8.9)$ & $10(10.4)$ \\
\hline There are no alternatives to injections in most cases & $17(34)$ & $9(19.6)$ & $26(27.1)$ \\
\hline Attitude of prescribers increase injection drug use & $30(60)$ & $46(100)$ & $76(79.2)$ \\
\hline
\end{tabular}


tion. In addition, conventional drugs are still widely used in the hospital despite the advent of atypical medications. The conventional marketed and supplied by independent drug suppliers are cheaper, easily available and affordable. Compared to atypical medications, they are rarely promoted or advertised by pharmaceutical sales representatives. In addition no pharmaceutical company can lay claim to a popular brand of the drug. The percentage reported in this study is, however, lower than the study in Zimbabwe which reported 94\% generic prescription. ${ }^{20}$ Prescribing in generic helps to reduce confusion while dispensing, improve control on drug inventory, prevent sudden out-of-stock or non-availability of drugs and makes drug purchase easier and more affordable to the patient. Although the hospital has experienced introduction and promotion of new atypical drugs marketed by large pharmaceutical companies, the practice of prescribing in generic has persisted. Reports from the present study also indicate that over a third of prescriptions were in injection form. While this percentage is comparable to the rate of $25 \%$ reported in a study in Syria, ${ }^{21}$ other studies have reported higher rates. Studies in Nepal, Lagos and Jordan reported $2.9 \%, 13.8 \%$ and $15 \%$ respectively. $1,2,3$ The high rate of injection use reported in present study can be attributed to the use of Depot medications in patients suffering from psychiatric illness. These medications are mainly used to improve adherence to medications although they are sometimes used as adjunct to oral drugs. There is also a high rate of parenteral drug administration in the treatment of acutely ill patients especially after a relapse. Some patients may present at the out-patient clinic in a relapsed state after defaulting from clinic appointment and refusal to comply with medications for a long period of time.

Most health care professionals attributed the practice of poly-pharmacy in the hospital clinics to the pressure and demands of patients who request for inclusion of certain medications in their prescription. This response is similar to the finding of the study of professional practices and perception. Bazigha et al. reported that a substantial number of physicians agreed that most prescriptions were patient-driven. ${ }^{2}$ Regarding prescription of generic drugs, majority of prescribers and dispensers attributed non-generic prescription to the influence of pharmaceutical companies who mount immense pressure on prescribers to prescribe their brands. This finding is corroborated by another study which reported that a substantial proportion of physicians agreed that their prescriptions were influenced by pharmaceutical representatives..$^{22}$ It is pertinent to note that a high proportion of the respondents especially dispensers agreed that many prescribers lacked required training in generic prescription. While it is true that medical curriculum strictly apply generic name teaching, individual practice of prescribers may be influenced by extraneous factors such as type of facility, role of pharmaceutical companies, volume of patients and potential financial benefits accruable to prescribers when a particular brand is prescribed. However, the role of continue medical education, training and awareness campaign among health care professionals cannot be overemphasized.

Although prescribing patterns in the hospital largely conforms to the concept of rational drug use, rational drugs use practices need to be improved in the areas of number of drugs prescribed per encounter and the percentage of drugs prescribed in injection form.

\section{Conclusions}

In conclusion, prescribing practices in the hospital were largely in compliance with rational drug use. However, improvements are required in the areas of poly-pharmacy and generic drug prescription. The relatively high number of drugs per encounter has been attributed to heavy work load at the outpatient clinic and the influence of patients on prescribers. Although generic prescription is above average, influence of pharmaceutical sale representatives was identified as a reason for the relatively low rate compared to similar studies conducted elsewhere.

We recommend that concerted efforts should be made to improve rational drug use practices in hospitals in Nigeria. Regular training programmes should be organized for health care professionals to promote the concept and practice of rational drug use. Educational programmes aimed at improving awareness among patients on the importance of rational drug use should be designed and implemented. Finally, government should formulate and implement regulations aimed at controlling the activities of pharmaceutical sale representatives.

\section{Limitations}

The study was conducted in a single facility. A multicentre study which allows comparison of drug use practices in many facilities would have produced results which can be more generalized. Furthermore, exclusion of patient indicators and facility indicators in the study design essentially deprived the study of important aspects of rational drug use.

\section{References}

1. Odusanya 00. Drug use indicators at a secondary health centre facility in Lagos, Nigeria. J Commun Med Primary Health Care 2004;16:21-4.

2. Bazigha AK, Fahmyn SA, Abu Gharbieh EF, Ali HS. Professional practices and perception towards rational use of medicine according to WHO methodology in United Arab Emirates. Pharmacy Pract 2006;8:70-6.

3. Alam K, Mishra P, Prabha M, et al. A study of rational drug prescribing and dispensing in outpatient in a tertiary care teaching hospital of Western Nepal. Kathmandu University Med J 2006; 4:436-43.

4. Khor M. Irrational drug use causing rise of anti-microbial resistance. World Health Assembly. The South-North Development Monitor (SUNS); 2004.

5. World Health Organization. Promoting rational use of medicines: Core components. WHO Policy perspectives on medicines. No. WHO/EDM/2002.3. Geneva: World Health Organization; 2002.

6. World Health Organization. The rational use of drugs: a report of the conference of experts. Nairobi, Geneva 1985, 25-9. Geneva: World Health Organization; 1987.

7. Mnyika KS, Killewo JZ. Irrational drug use in Tanzania. Health Policy Plan 1991;6:182-4.

8. Kuruvilla K. Rational drug therapy in psychiatry: a doctor's approach. Rational Drugs 2006;24.

9. de Menil V. Rational use and rationale for use: Psychiatric medication at an Argentine Institution for intellectual disability. Transcult Psychiat 2009; 46:651-71.

10. World Health Organization. How to investigate drug use in health facilities: selected drug use indicators. Geneva: World Health Organization; 1993.

11. Mouala C, Abeye J, Somse P, et al. Prescribing and dispensing generic drugs in the Mambere-Kadei Health District of the Central African Republic. Med Trop (Mars) 2008;68:149-54.

12. Hazra A, Tripathi SK, Alam MS. Prescribing and dispensing activities at the health facilities of a non-governmental organization. Natl Med J India 2000;13:177-82.

13. Bosu WK, Ofori-Adjei D. A One day survey of prescribing patterns in the District General Hospital of the Wassa West District of Ghana. Tropical Doctor 1997;27:222-6.

14. Victoria CG, Facchini LA, Grassi-Filho M. Drug usage in Southern Brazillian hospitals. Tropical Doctor 1982;12:231-5.

15. Maggiore RJ, Gross CP, Hurria A. Polypharmacy in older adults with cancer. Oncol Express 2010;15:507-22.

16. Department of Health. Polypharmacy and older people. Nurses Times Net 
2003;99:54.

17. Flaherty JH, Perry II HM, Lynchard GS, Morley JE. Polypharmacy and hospitalization among older home care patients. J Gerontol Med Sci 2000;55A:554-9.

18. Wilson K. Polypharmacy: Making it all work. 10th Conference on Polypharmacy; 2010.

19. Russell PS, George C, Mammen P. Predictive factors for polypharmacy among child and adolescents psychiatry inpatients. Clin Pract Epidemiol Mental Health 2006;2:25.

20. Hogerzeil HV, Ross-Degnan D, Laing RO, et al. Field tests for rational drug use in twelve developing countries. Lancet 1993;342:1408-19.

21. Ottom SA, Sequeira RP. Health care providers' perceptions of the problems and causes of irrational use of drugs in two Middle East Countries. Int J Clin Pract 2006;60:565-70.

22. Biswas NR, Biswas RS, Pal PS, et al. Patterns of prescription and drug use in two tertiary hospitals in Delhi. Indian J Physiol Pharmacol 2000;44:109-12. 